\title{
Comparison of Polarization Bidirectional Reflectance Distribution Function (BRDF) Models
}

\author{
Michael Hess \\ Richard Priest \\ US Naval Research Laboratory (Code 5620) \\ 4555 Overlook Ave., S.W. \\ Washington, D.C. 20375
}

\begin{abstract}
IR target signature models rely on some representation of the BRDF to describe surface optical properties. For unpolarized signature modeling, it usually suffices to split the BRDF into a diffuse part and a specular part, and to model each part with a few parameters fit from data; the Sandford-Robertson approach is perhaps the bestknown. Artifacts produced by this idealization are almost always small compared with true signature features. For polarization modeling, however, one computes quantities, such as the degree of polarization, which are small differences of large numbers, and which derive from small differences between elements in the representation of the polarization BRDF. An adequate representation is difficult to determine because the conventional scalar BRDF function generalizes to a matrix function with typically 6-10 independent elements, requiring that numerous parameters be determined from data in a consistent manner. This requires a new BRDF representation which is based on physical principles, and which is comprehensive and accurate enough to satisfy requirements for use in signature modeling. This paper considers several candidate schemes for parameterizing the polarization BRDF from the standpoints of consistency and the ability to model all important features of the surface optical properties, without introducing so many parameters as to be ill-conditioned.
\end{abstract}

\section{TABLE OF CONTENTS}

1. INTRODUCTION

2. BRDF MEASUREMENT AND REPRESENTATION

3. POLARIZATION BRDF MODELING APPROACH

4. CONSTRUCTION OF THE PARAMETRIC MUELler MATRIX

5. CONCLUSIONS

6. ACKNOWLEDGEMENT

7. REFERENCES

\section{INTRODUCTION}

The polarization attributes of target and background optical signatures, particularly in the thermal infrared bands, are of interest for a variety of applications such as enhancing detection of targets in cluttered backgrounds, and deducing the optical properties of remotely sensed surfaces. A highquality optical signature model is essential to support the development of techniques for exploiting Polarization Information (PI). Uses for such a model include:

U.S. Government work not protected by U.S. copyright. generalizing from the few specific cases for which one can afford to collect data to a wide spectrum of environmental conditions and sensor characteristics; predicting the effects of changes in such target characteristics as surface optical properties, geometry, and operating condition; and addressing test and analysis efforts to the most critical phenomenology issues.

The Naval Research Laboratory, under a Central MASINT Office (CMO)-led effort to explore the utility of PI, is tasked with identifying and/or developing, and validating, a state-of-the-art IR polarization signature model to support data collection and analysis, and performance prediction. An industry survey concluded that, while no extant code represented polarization phenomenology with the needed fidelity, the modeling task should proceed by upgrading an existing code rather than undertaking the development of a new code. The survey identified three signature codes that appear to be good candidates for such an upgrade:

- IRMA[1] - This code was developed by Nichols Research Corp., Shalimar, FL, largely under the sponsorship of AF Research Lab, Eglin AFB. While the current distribution version (4.1) has been validated for non-polarization cases, it contains a very simple BRDF representation, and does not adequately handle polarization effects. Recently, a pre-release version of IRMA which has full Stokes vector/Mueller matrix capabilities, and hence is nominally able to handle linear and circular polarization, was received by NRL and is under evaluation.

- OPTASM[2] - This code was developed by Acquista and Rosenwald of PAR Government Systems Inc. In 1997, support for the code was taken over by Surface Optics Corporation (SOC) of San Diego, CA, a company long associated with the characterization of surface optical properties. The current version (2.1.23), which has been available for several years, contains a full polarization representation. Validation of OPTASM's polarization signature capabilities has been hampered by the absence of a detailed polarized BRDF data base - a situation now being rectified, as discussed below.

- $\quad$ SPIRITS-Pol[3] - The basic (unpolarized) SPIRITS code was developed by Aerodyne Corporation of Billerica, MA, with multi-Service support led by AF Research Lab, Hanscom AFB. SPIRITS has achieved "DoD-standard" status through the mechanism of JANNAF. A recently received polarization variant of this code developed under Navy sponsorship is being 
used in this work; when (and if) it is validated, this version may be issued as JANNAF-standard version AC3.

The thrust of NRL's effort under the CMO program, then, is to: determine a represention of the polarized reflection and scattering properties of surfaces which is suitable for signature modeling purposes; arrange for the collection of a (small) database of polarized optical properties for a few representative surface materials; incorporate the optical data into the candidate signature models by adapting the selected BRDF representation appropriately; and employ the best resulting model in support of the CMO program.

\section{BRDF MEASUREMENT AND REPRESENTATION}

The basic approaches of the three candidate codes are similar. Each represents targets as collections of planar surfaces or "facets". Optical properties of each facet are defined with an appropriate level of complexity, depending, for example, on whether the polarized signature is required, or on whether the reflectance behavior varies with rotation of the surface around the surface normal. Reflection of radiation incident on the target from its surroundings, generally characterized as "skyshine" and "earthshine" (with possibly a few "point sources" such as the sun) is modeled. Shadowing of one part of the target from the background by another is usually accounted for with varying degrees of sophistication, as is reflection of one part of the target by another. The temperatures of the target's surfaces are calculated by balancing the radiative, convective, and conductive heat fluxes. Finally, the radiance of each (visible) facet in the direction of the sensor is calculated as the sum of the emitted and reflected components.

Models of this type have been used extensively to predict the total (i.e. unpolarized) radiance of many types of targets. With suitable attention to detail, including use of a fairly detailed representation of surface optical properties, agreement of model predictions of radiance with measured data to within better than $20 \%$ has been obtained. The function used to describe the directional dependence of reflected energy is the BRDF. The geometry of the BRDF definition is shown in Figure 1.

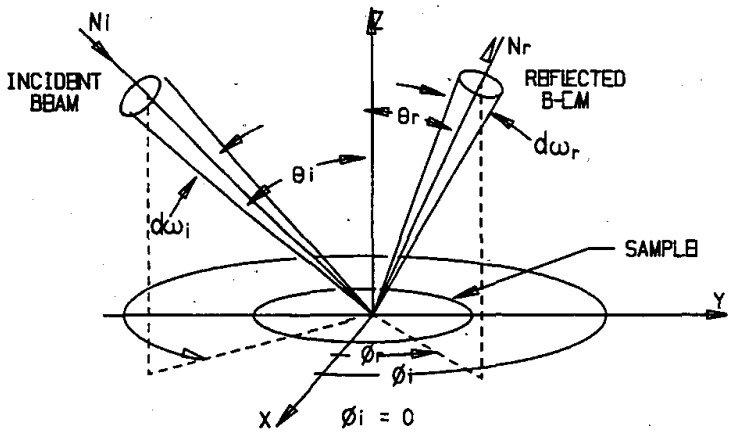

Figure 1 BRDF Geometry
The BRDF is defined as the ratio of the reflected radiance $\left(\mathrm{w}-\mathrm{m}^{-2}-\mathrm{sr}^{-1}\right)$ in a particular direction $\left(\theta_{\mathrm{r}}, \phi_{\mathrm{r}}\right)$ to the incident irradiance $\left(w-m^{-2}\right)$ from direction $\left(\theta_{i}, \phi_{i}\right)$.

$$
f\left(\theta_{i}, \phi_{i} ; \theta_{r}, \phi_{r}\right)=\frac{N_{r}\left(\theta_{r}, \phi_{r}\right)}{I_{i}\left(\theta_{i}, \phi_{i}\right) \cos \theta_{i}}
$$

The units of the BRDF are inverse solid-angle $\left(\mathrm{sr}^{-1}\right)$. In this paper, we treat the case of a surface with no preferred azimuth orientation (e.g. no directional features or striae); for this case, $\phi_{\mathrm{i}}$ or $\phi_{\mathrm{r}}$ may be taken to be zero, and BRDF is a function of three angles: $\theta_{\mathrm{i}}, \theta_{\mathrm{r}}$, and the azimuth angle between the incident and reflected rays, $\phi$.

Figure 2 shows a pictorial representation of a typical BRDF, taken from the well-known paper by Nicodemus[4] that provides a good physical description of these concepts.

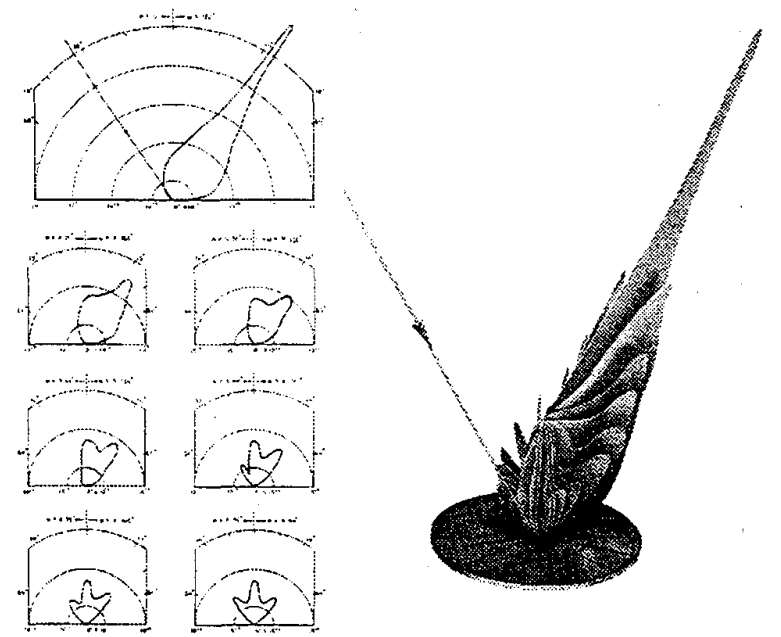

Figure 2 BRDF Visualization

The integral of the BRDF over all reflected angles provides the dimensionless directional hemispherical reflectance (DHR), $\rho(\theta)$. (Similarly, the integral of the BRDF over all incident angles gives the hemispherical directional reflectance (HDR). Because the BRDF is invariant under interchange of incident and reflected angles (reciprocity) the HDR and DHR are equivalent, and can be used interchangeably.)

Accurate BRDF measurements for signature analysis require a systematic mapping of the light scattered into the hemisphere. Figure 3 shows SOC's Model 200 Bidirectional Reflectometer, used for performing laboratory BRDF measurements.

This fully automated instrument provides full hemispheric coverage for both unpolarized BRDF and Mueller matrix measurements. Light sources for the measurements include quartz halogen and $1700 \mathrm{C}$ blackbody broadband sources, and up to five optional laser sources. A number of detectors are employed, e.g., photomultiplier tube, lead sulfide, mercury cadmium telluride, to provide continuous spectral coverage from 0.3 to 14 microns. Wavelength selection is 
accomplished by inserting thin film bandpass filters into the optical train in front of the detectors. The noise floor of the instrument is $10^{-3}$ ster $^{-1}$ for broadband sources and $10^{-6}$ ster $^{-1}$

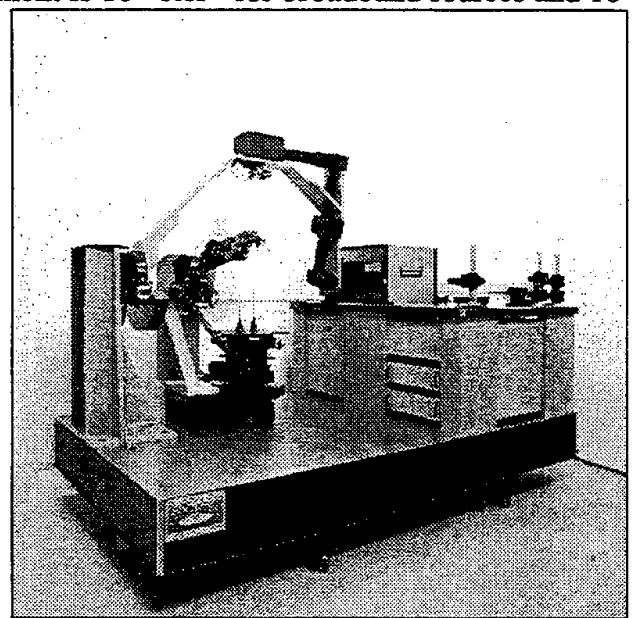

Figure 3 SOC-200 Bidirectional Reflectometer

for laser sources. A paper by Beecroft, et al[5] provides a detailed description of the capabilities of this instrument. Figure 4 shows an example 3-D plot of the unpolarized BRDF of a painted surface at a wavelength of 0.5 microns and $50^{\circ}$ incident angle.

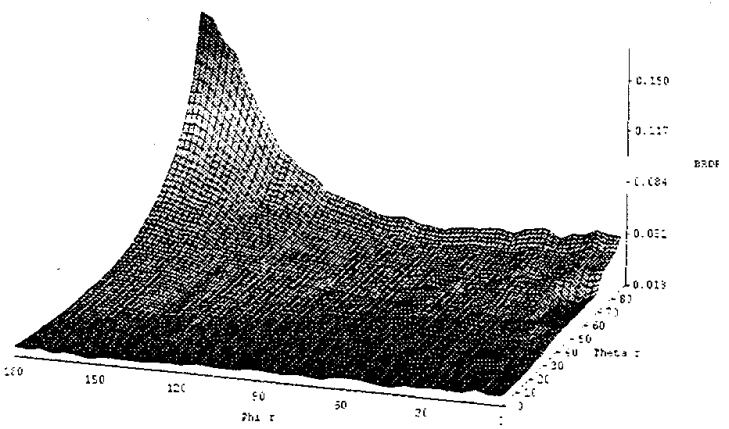

Figure 4 Unpolarized BRDF of Painted Surface

SOC is currently upgrading the Model 200 for making polarization BRDF measurements by incorporating the setup shown in Figure 5. With the planned complement of sources, measurements will be made at $0.488,0.6328$, $1.064,3.39$, and $10.6 \mu \mathrm{m}$. Manipulation of the polarization generating and analyzing components in accordance with well-established techniques allows derivation of the Mueller matrix from the raw measurements.

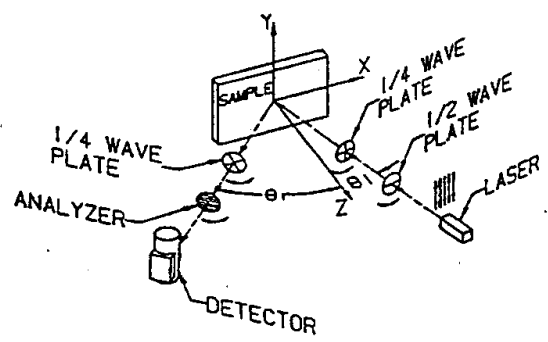

Figure 5 Setup for Mueller Matrix Measurement
An essential element of the Mueller matrix data processing will be the development of measurement standards. SOC has developed laser reflectance measurement standards for laser sources in the past. By use of the standards, the values within the Mueller matrix may be reported in units of inverse steradians $\left(\mathrm{sr}^{-1}\right)$, as needed for the calculation of target signatures.

The ideal standard must be nearly diffuse at the wavelength of interest, and must have strongly cross-polarizing characteristics. Measurements made by Azzam and Giardina[6] for an aluminum-coated grating indicate very desirable features for the development of a standard at 10.6 $\mu \mathrm{m}$ and other laser wavelengths. SOC has developed processes for generating gratings by photofabrication, and is currently operating equipment capable of constructing of a Mueller matrix standard.

It will be necessary to collect optical properties data for each material of interest. One could simply apply these data in table lookup fashion for signature modeling - while megabytes of storage would be needed for each material, today's processors make this approach feasible. Nevertheless, there are several motivations for developing a physically-based parametric BRDF representation for the polarized case, with which the measured data can be reviewed, interpreted, and abstracted:

- As with any large-scale data collection, means must be found to screen data for reasonableness before they are reported and used in modeling. Using the collected data to instantiate a parametric BRDF representation provides a framework for screening and assessing the quality of the data. In particular, since emissivity is not measured directly, but is inferred from reflectance measurements, development of a BRDF model will allow direct checking and constraint of the Mueller matrix to ensure consistency with the laws of thermodynamics.

- The parametric representation can help with the "dynamic range" problem that results from the model's need for high angular resolution around the specular lobe, but lower resolution elsewhere. A BRDF model can inform the process of using data from high resolution sampling of the lobe, gathered at a few key angles, to interpolate among lower resolution samples taken over the full volume.

- Measured reflectance data are chronically unreliable near grazing incidence, for practical instrumentation-related reasons. A BRDF model can help choose reasonable values in such regions.

- The process of developing, instantiating, and validating a BRDF model will inevitably force attention to the underlying mechanisms of coating performance, thereby improving knowledge and awareness of those principles, which are now rather poorly understood.

\section{POLARIZATION BRDF MODELING APPROACH}

For signature modeling, an operational definition of the BRDF function, $f$, is:

$$
R\left(\theta_{o}\right)=\iint \sin \theta_{i} d \theta_{i} d \phi \cdot R\left(\theta_{i}, \phi\right) \cdot f\left(\theta_{o}, \theta_{i}, \phi\right) \cdot \cos \theta_{i}
$$


where $R\left(\theta_{i}, \phi\right)$ is the incident and $R\left(\theta_{0}\right)$ the reflected radiance. It is possible to make some simplifying idealizations of the BRDF representation for unpolarized modeling without excessive error. The most common approximation is to assume that reflection and scattering can be represented as the superposition of ideal specular and diffuse terms. In the case of ideal specular reflectance, $\mathrm{f}$ is a delta function at the specular angle. The integration is straightforward to carry out resulting in:

$$
R_{o}(\theta)=\rho(\theta) \cdot R_{i}(\theta)
$$

Thus, for ideal specular reflection, determination of the DHR amounts to determination of the BRDF. For the diffuse reflectance, the modeling approach is to replace $R\left(\theta_{i}\right)$ in Eq. 1 with its average over the hemisphere. This replacement tacitly assumes that this component of reflectance is sufficiently non-directional (and/or that there is so little structure in the incident radiance) that the angular dependence of the incident light can be ignored. The integral again becomes straightforward, with the result:

$$
R_{o}(\theta)=\rho(\theta) \cdot R_{\text {avg }},
$$

where $R_{a v g}$ is the incident radiation field averaged over the hemisphere. Again note that the BRDF corresponds to the DHR, and like $\rho$ is a function of only one angle for the combined specular and diffuse components.

Extension of this simplified approach for use with polarized radiation will now be considered for illustration, although refinements will be found to be necessary. The form of the BRDF changes for calculations involving polarized radiation. The four-component Stokes vector[7], which completely describes the time-averaged polarization state of incoherent light, replaces the usual scalar radiance. For the polarized case, the BRDF takes the form of a Mueller matrix[8], a $4 \times 4$ matrix which relates the Stokes vector of reflected light to that of incident light, so Eq. 2 or Eq. 3 generalizes to:

$$
S_{o}=M S_{i}
$$

Under the usual convention, the elements of $\mathbf{S}$ are denoted as $S_{0} \rightarrow S_{3}$. Note that $\mathbf{S}$ doesn't behave as a normal vector; under coordinate rotations, for example, only the linear polarization elements, $S_{1}$ and $S_{2}$, are mixed with each other. As the DHR gave the BRDF for the unpolarized case, determining the Mueller matrix in Eq. 4 is tantamount to specification of the BRDF for the polarized case:

As mentioned, observed target surface radiances are the combinations of the reflected and emitted components. As with the non-polarization case, reflectivity and emissivity are related through Kirchhoff's law. The generalization of this law to the polarization case involves replacing scalar emissivity by a four-component vector, $\mathbf{E}$. The product of $\mathbf{E}$ with Planck's blackbody function is the Stokes vector describing the emitted radiation. Symmetry considerations help determine how Kirchhoff's law must be generalized for polarization. It is clear that $E_{3}$, which describes the preference for right circular over left circular polarization, must be zero by symmetry. The most useful coordinate system for this discussion is the one defined by the principal plane containing the surface normal and the direction of emitted radiation. In this "preferred" coordinate system, the $z$-axis is perpendicular to the surface, the $x$-axis is in the principal plane, and the $y$ axis is normal to the principle plane. In this coordinate system, symmetry also ensures that $E_{2}$ is zero.

$E_{0}$ and $E_{1}$ can be determined by considering the simple holraum scenario, in which the object being modeled is inside a closed chamber with black walls at the temperature of the object. To satisfy the first and second laws of thermodynamics, the sum of the emitted and reflected radiance emerging from a surface of the object must be unpolarized and equal to the Planck blackbody radiance.

The output Stokes vector, $S_{\circ}$ of Eq. 4, is unpolarized and is thus a column vector proportional to $(1,0,0,0)^{\mathrm{T}}$. Kirchhoff's law can then be stated as:

$$
\boldsymbol{M}\left(\begin{array}{l}
1 \\
0 \\
0 \\
0
\end{array}\right)+\left(\begin{array}{c}
E_{0} \\
E_{1} \\
0 \\
0
\end{array}\right)=\left(\begin{array}{l}
1 \\
0 \\
0 \\
0
\end{array}\right)
$$

where Planck's function has been factored out. The Mueller matrix in this equation is the sum of diffuse and specular parts. This vector equation can be written as 4 ordinary equations:

$$
\begin{aligned}
& M_{0,0}+E_{0}=1 \\
& M_{1,0}+E_{1}=0 \\
& M_{2,0}=0 \\
& M_{3,0}=0
\end{aligned}
$$

The first of these is recognized as the usual form of Kirchhoff's law. The second determines $E_{1}$ in terms of $M_{1,0}$. The third and fourth equations constrain $\mathbf{M}$, but this is not a problem because, in the preferred coordinate system, these elements are zero by symmetry. The apparent asymmetry of the second equation is not a problem either, since symmetry considerations force $\mathbf{M}_{1,0}=\mathrm{M}_{0,1}$ in the preferred coordinate system. In terms of angular dependence, $\mathbf{E}$ is obviously a function only of the angle of emission from the surface normal, for the assumed case of no preferred azimuth orientation.

A final note concerns how the Stokes vectors and $\mathbf{E}$ transform under rotations from the preferred coordinate systems (which is typically a local coordinate system for each model facet) to the global coordinate system (which is usually specified by $z$ being the vertical and $x$ lying in the plane defined by the vertical and the observation direction). This transformation can be written as $S \rightarrow T S$, where $T$ is of the form: 


$$
\left[\begin{array}{cccc}
1 & 0 & 0 & 0 \\
0 & \cos (2 \alpha) & \sin (2 \alpha) & 0 \\
0 & -\sin (2 \alpha) & \cos (2 \alpha) & 0 \\
0 & 0 & 0 & 1
\end{array}\right]
$$

where $\alpha$ is the angle of rotation about the facet normal which would carry the local $\mathrm{x}$ axis into the plane defined by the vertical and the direction to the observer. As mentioned above, only the second and third components of Stokes vectors and $\mathbf{E}$ are mixed by this transformation. Transformation properties of $\mathbf{M}$ under coordinate rotations can be inferred from the form of $\mathbf{T}$ given above.

The discussion to this point has focused implicitly on the contributions of reflections of area sources, such as the sky. The specular reflection of point sources results in glints which, in facetized model calculations, must be handled by specialized approaches. On the other hand, diffuse reflection of point-like sources, such as the sun, can be fit into the formulation described above. Following the replacement used in Eq. 3, a normally incident point source irradiance, $I_{i}$, must be replaced by an equivalent incident radiance. If the source is taken as a a Lambertian emitter which fills the upper hemisphere, then the effective radiance is $R_{\text {avg }}=\mathrm{I}_{\mathrm{i}} / \pi$. If the point source is incident off-normal, a factor of $\cos \left(\theta_{i}\right)$ must be included. The approach then is to identify $R_{a v g}$ as $\left(I_{i} / \pi\right) \cos \left(\theta_{i}\right)$. Some refinements to this idea will be discussed below. The point of this paragraph is to note that for point sources, as for area sources, it suffices to consider in detail only the Mueller matrix - the generalization of the DHR.

\section{CONSTRUCTION OF THE PARAMETRIC MUELLER MATRIX}

A step-by-step approach is taken to highlight the issues involved in constructing a parametric Mueller matrix. The discussion relates directly to IRMA and SPIRITS-Pol, which use a model-based BRDF representation. It is less applicable to OPTASM, which relies on numeric fits to a BRDF data base; as discussed in the introduction, however, a successful model-based representation will inform the use of data in OPTASM as well. We start with a case - specular Fresnel reflectance from a thick dielectric surface - for which the exact result is known.

\section{Ideal Specular Reflectance Only}

In Fresnel reflectivity, an incident field is partially reflected and partially transmitted at the planar boundary between two dissimilar dielectric materials (the first of which is usually air.) If the second dielectric is much thicker than $\lambda /$, where $\lambda$ is wavelength and $\kappa$ is the imaginary part of the index of refraction, the transmitted part can be ignored and the Mueller matrix constructed from the Fresnel reflectivity. The Mueller matrix has the form:
$\boldsymbol{M}=\boldsymbol{F}=\frac{1}{2}\left(\begin{array}{cccc}\rho_{s} \rho_{s}^{*}+\rho_{p} \rho_{p}^{*} & \rho_{s} \rho_{s}^{*}-\rho_{p} \rho_{p}^{*} & 0 & 0 \\ \rho_{s} \rho_{s}^{*}-\rho_{p} \rho_{p}^{*} & \rho_{s} \rho_{s}^{*}+\rho_{p} \rho_{p}^{*} & 0 & 0 \\ 0 & 0 & \rho_{s} \rho_{p}^{*}+\rho_{p} \rho_{s}^{*} & -\left(\rho_{s} \rho_{p}^{*}-\rho_{p} \rho_{s}^{*}\right) \\ 0 & 0 & \left(\rho_{s} \rho_{p}^{*}-\rho_{p} \rho_{s}^{*}\right) & \rho_{s} \rho_{p}^{*}+\rho_{p} \rho_{s}^{*}\end{array}\right)$

where $\rho_{\mathrm{s}}$ and $\rho_{\mathrm{p}}$ are Fresnel amplitudes which depend on the angle between the outgoing radiation and the surface normal. Standard references give the functional form of $\rho_{s}$ and $\rho_{p}[9]$; of interest here is the general structure. Note that $\mathbf{M}_{0,1}=\mathbf{M}_{1,0}$ and $\mathbf{M}_{2,0}=\mathbf{M}_{3,0}=0$ as required above. The form obeys the most restrictive of the symmetry types described by van de Hulst[10]. In this form there are two parameters, the real and imaginary parts of the index of refraction, $n$ and $\kappa$, which enter into the formulas for the Fresnel amplitudes. If this form is used for $\mathbf{M}$, and $\mathbf{E}$ is calculated from Eq. 6, a consistent and physically correct model is assured.

In addition to its modeled BRDF approach, the IRMA code allows a Mueller matrix to be entered directly into a database file, typically for about 10 different scattering angles. Thus, all that is required to use this approximation with IRMA is to select values for $n$ and $\kappa$, use Eq. 7 to calculate the matrix elements for an number of angles, and enter the results into a file.

The SPIRITS-Pol model takes a somewhat different approach based on the Sandford-Robertson (SR) parametric model[11], [12], which provides a number of advantages. The SR model has 4 parameters which are determined so as to fit the model to measured data; they are:

- diffuse spectral reflectance coefficient, $\rho_{D}(\lambda)$, which describes the magnitude of the diffusely reflected component as a function of wavelength,

- $\quad$ spectral total hemispherical emissivity, $\varepsilon_{0}(\lambda)$, which is determined as the complement of total reflectivity as a function of wavelength, and

- two parameters which are independent of wavelength, and which describe the increase of reflectivity near grazing incidence (b), and the specular lobe width (e).

For the purpose of this section two of these parameters, $\rho_{D}$ and $e$, are taken to be zero. The SR model focuses on the directional emissivity, the $\mathrm{E}_{0}$ of Eq. 5 . In SR it is given by:

$$
\begin{aligned}
& E_{0}=\varepsilon_{0} \cdot g(\theta) / G \\
& g(\theta)=\frac{1}{1+b^{2} \tan ^{2}(\theta)} \\
& G=\frac{1}{1-b^{2}}\left(1+\frac{1}{1-b^{2}} \log \left(b^{2}\right)\right)
\end{aligned}
$$

$\mathrm{G}$ is a normalization factor which ensures that the cosine weighted angular average of the emissivity is equal to $\varepsilon_{0}$. The parameters $b$ and $\varepsilon_{0}$ control the angular form and strength of the emissivity. To observe the constraint of the first part of Eq. 6, SPIRITS-Pol takes $\mathbf{M}$ to be a scaled Fresnel form equal to $\mathrm{k}_{\text {ess }}(\theta) \mathbf{F}$, with $\mathbf{F}$ given by Eq. 7 and 


$$
k_{e s s}(\theta)=\frac{1-\varepsilon_{0} g(\theta) / G}{F_{0,0}(\theta)}
$$

Since the scaled form of $\mathbf{F}$ applies to all its elements, the form for $E_{1}$ is

$$
E_{1}=-k_{e s s} F_{0,1}(\theta)
$$

The latter two elements of $\mathbf{E}$ are zero as above. The motivation for this approach is that, given a choice for $\varepsilon_{0}$ and $b$, one can often choose $n$ and $\kappa$ such that $k_{\text {ess }}$ is very close to unity, and weakly dependent on $\theta$. When this is the case, the formulation is very nearly that of Fresnel and, as will be seen below, also allows some useful generalizations.

\section{Ideal Specular and Ideal Lambertian Diffuse Reflectances}

The first enhancement to the ideal specular model is the addition of an ideal Lambertian diffuse reflectance. For this component, the reflected radiation is proportional to the projected area (cosine law) and unpolarized. This means that $\rho$ is independent of angle, and that $\mathbf{M}$ has just one nonzero element $-M_{0.0}$, which also is angle independent.

The IRMA model provides for specification of a diffuse reflective contribution which is additive to the $\mathbf{M}_{0,0}$ element. $\mathbf{E}$ is calculated from Eq. 6 as above, and IRMA does not allow angular dependence for the diffuse part of the reflectance. One problem with this approach is that if a diffuse contribution is used, care must be taken with the specular contribution lest $\mathbf{M}_{0.0}$ become greater than 1 for angles near grazing (where $F_{0.0}$ is near 1.) Since the specular Mueller matrix can be arbitrarily specified, this problem can be avoided by an ad hoc tempering of $\mathbf{F}$; however, there is no physical model to guide this procedure.

SPIRITS-Pol takes an approach similar to that of IRMA in that a diffuse reflectivity is modeled as an addition to $\mathbf{M}_{0,0}$. However, unless the $b$ parameter is set to zero, the diffuse reflectivity will be angle dependent. In the $S R$ model as used in SPIRITS-Pol, the diffuse reflectivity enters the formalism in a manner similar to emissivity. If the diffuse reflectivity strength, $\rho_{D}$, is chosen to equal $\left(1-\varepsilon_{0}\right)$, and $b$ is set to zero, the model describes an ideal Lambertian reflector. The mechanism for this is that the strength of the specular reflection is essentially $1-\left(\rho_{D}+\varepsilon_{0}\right)$. However, if $\rho_{D}$ is less than $\left(1-\varepsilon_{0}\right)$, the Mueller matrix becomes:

$$
\begin{aligned}
& M_{i, j}=k_{e s s} F_{i, j}+\left(\rho_{D} g(\theta) / G\right) \delta_{i, 0} \delta_{j, 0} \\
& k_{e s s}=\left[1-\left(\rho_{D}+\varepsilon_{0}\right) g(\theta) / G\right] / F_{0,0}
\end{aligned}
$$

If $b$ is chosen to be zero, there is no choice for $n$ and $\kappa$ which will allow $k_{e s s}$ to be near unity for all angles, which can lead to nonphysical results. SPIRITS-Pol is thus not well-suited to modeling a mixture of ideal Lambertian plus ideal Fresnel specular behavior.

\section{Ideal Specular Plus General Diffuse Reflector}

Data suggest that most painted surfaces are not ideal Lambertian reflectors. In particular, the total hemispherical reflectivity is angle dependent, which was one of the original motivations for the SR model in which the diffuse reflectance is allowed angular dependence.

The IRMA model does not allow for angle dependence of the diffuse contribution. It would not be difficult to extend it to allow this case, but the ad hoc procedure for preventing elements from becoming greater than unity would remain.

On the other hand, SPIRITS-Pol is well-suited to this case. As above, $n$ and $\kappa$ must be chosen to be compatible with the parameter $b$. Once this is done, any desired admixture of specular and diffuse character can be obtained by adjusting $\rho_{D}$. A further refinement concerning the treatment of point source reflectance will be discussed in the next section.

\section{General Specular Reflectance}

Data show that typical weathered material surfaces are never ideal specular reflectors - even the glossiest surfaces exhibit some angular width of the glint lobe. This was the motivation for the modeled lobe width function and related glint lobe parameter, $e$, in the SR model. This part of the SR model is based on the work of Trowbridge and Reitz[13]. In this approach, the surface is regarded as an ensemble of microfacets (sometimes called "sequins"), distributed in orientation. Reflection from each microfacet is ideal specular, but since there is a distribution of facet orientations about the surface normal, radiation from a point source is reflected into a glint lobe with width controlled by the microfacet angular distribution. This mechanism generates out-of-plane scattering.

The general formulation of the IRMA model does not allow for glint lobe width. However, a specialized (unpolarized) treatment is provided for reflection of the sun.

The SPIRITS-Pol model incorporates the SR e parameter and associated model mechanism for point source reflection. SPIRITS-Pol assumes that a microfacet normal bisects the source and observation directions in this connection. This allows the specular part of the full Mueller matrix to be used to calculate a polarized scattering contribution. If the e parameter is chosen to represent a large glint width, this can simulate a polarized diffuse reflectance; however, this mechanism is limited to point sources. A further limitation concerns the constrained angular dependence of the SR model.

Since a non-zero glint lobe width allows out-of-plane scattering, the DHR can not fully determine the polarization properties of the BRDF. A significant problem concerns the definition of the preferred coordinate system, as there are three non-coplanar directions involved. The idealization of a microfacet normal which bisects the source and observation directions neatly resolves this problem, but there are significant practical problems in applying this idea 
to specular reflections of extended objects (which may be why it is not employed in either IRMA or SPIRITS-Pol.)

\section{General polarized diffuse reflectance.}

One mechanism for obtaining polarized diffuse reflectance was discussed above. Another mechanism, dealing with volume scattering in paints, was suggested by Maxwell[14] several years ago. In this mechanism, the paint is viewed as a binder in which pigment particles are distributed at random. The surface of the paint is viewed as a planar binder/air interface. Incoming radiation is partially transmitted into the paint where it is randomized and depolarized by multiple scattering from pigment particles. Some fraction of the (now unpolarized) energy exits the binder/air surface via Fresnel transmission at all angles, and radiation exiting at non-normal angles becomes polarized in accordance with Fresnel transmission behavior. This polarization mechanism is essentially the same as the one which polarizes thermal emission, as in Eq. 6. Currently, none of the polarization codes implements this idea. Since the mechanism is so closely related to thermal emission, it should be possible to incorporate it into the formulation described above.

\section{CONCLUSIONS}

IRMA and SPIRITS-Pol base their derivation of the polarization BRDF on determination of the DHR. In this, they can be regarded as being guided by the Fresnel reflection principal. Reliance on such a physics-based approach helps to ensure self-consistency. It further ensures that the generalized form of Kirchoff's law will correctly model self-emission. Finally, it is a simple approach with functional dependence on only a single angle variable. On the other hand, these models may not capture all polarization-related phenomena exhibited in real scenarios.

IRMA allows an arbitrary ideal specular reflectivity to be specified, and accommodates a very simple, unpolarized diffuse scattering contribution. In fact, freedom to modify the general form of the specular Mueller matrix is required to accommodate the diffuse contribution without introducing nonphysical artifacts. SPIRITS-Pol introduces a scaled form of the Fresnel Mueller matrix in order to accommodate the unpolarized SR model formulation for $\mathbf{M}_{0.0}$ and $\mathrm{E}_{0}$. This model compromise does not detract significantly from the advantages of the strictly Fresnel approach in most cases. It allows an angle dependent, but unpolarized, diffuse contribution to be modeled, as well as a general, polarized treatment of point source specular reflection. The IRMA and SPIRITS-Pol models are physically grounded in that they build on the Fresnel result. Thus, for materials which exhibit Fresnel-like behavior, good results can be expected.

The IRMA model cannot be easily generalized to cover nonideal specular reflection; thus, it does not deal with out-ofplane scattering and the related polarization properties. IRMA does allow a table form of the Mueller matrix, but provides no guidance on a consistent method of generalizing beyond the ideal Fresnel case. The SPIRITS-Pol model offers a more structured way to extend beyond the Fresnel case, but it is tied to the SR model approach. The idea of using a scaled Fresnel form is reasonable, but is an uncontrolled approximation that may not always work well. The SR-based approach allows a polarized out-of-plane scattering contribution, but only for point sources. Extension to extended sources, such as reflection of other model facets, would require extensive modification of the model.

OPTASM differs in that it does not focus on hemispherical reflectivity modeling, instead relying directly on measured data. By relying on numeric fits to a comprehensive, detailed set of BRDF measurements, it is likely to represent all polarization effects with some degree of fidelity. However, there is risk that without traceability to a wellfounded base case, errors in the data used to fit the many parameters in the BRDF model may lead to hard-to-catch artifacts. Artifacts are also possible from the assumed model functional form which is used to fit the data, a sum of Lorentzian forms. The model calculates a hemispherical reflectivity consistent with the numeric fits, and uses this hemispherical reflectivity to calculate the emissive contributions via Kirchoff's law. No testing has yet been conducted to assess the possible pitfalls of this approach, due to the absence of an adequate polarization BRDF data base. Thus, the physics-based approaches of IRMA and SPIRITS-Pol are logical (and instructive) starting points for this effort, but may ultimately prove inadequate. In this case, the more data-driven approach used by OPTASM may be preferable.

\section{ACKNOWLEDGEMENT}

The authors gratefully acknowledge the contribution to this paper by James Jafolla of Surface Optics Corporation of the text and figures on BRDF measurement.

\section{REFERENCES}

[1] IRMA Version 4.I Multi-Sensor Signature Prediction Model User's Manual, May 1998 (on distribution CD ROM).

[2] C. Acquista and R. D. Rosenwald, Software User's Manual for the Optical Target Signatures Model (OPTASM), Version 2.1, Apr. 1997.

[3] J. Conant, J. DeAngelis, D. Drugan, and M. Vaughn, The JANNAF Spectral and In-Band Radiometric Imaging of Targets and Scenes Model - Aircraft Version I (SPIRITSAC1)Vol. I, Sep. 1994

[4] F. Nicodemus, "Directional Reflectance and Emissivity of an Opaque Surface”, Appl. Opt., 4, pp. 767-773, 1965.

[5] M. Beecroft, J. Neu and J. Jafolla, "Bidirectional reflectance data to support paint development and signature calculations," Proceedings of the SPIE Symposium on Stray Radiation in Optical Systems II, vol. 1753, pp. 304-316, 1992. 
[6] R. Azzam and K. Giardina, "Conventional and Generalized Mueller-Matrix Ellipsometry using the FourDetector Photopolarimeter", Opt. Eng., 30, no. 10, pp. 15831588, 1991.

[7] W. Shurcliff, Polarized Light, Harvard University Press, 1962.

[8] Shurcliff, op. cit.

[9] M. Born and E. Wolf, Principles of Optics, Pergamon Press, 1986.

[10] H.C. van de Hulst, Light Scattering by Small Particles, New York: Dover, 1981.

[11] Conant, DeAngelis, Drugan, and Vaughn, op.cit.

[12] F. J. Iannarilli, Aerodyne Research, "Augmentation of SPIRITS and Sandford-Robertson BRDF for elliptical polarization," private communication.

[13] T. Trowbridge and K. Reitz, "Average irregularity representation of a rough surface for ray relection," J. Opt. Soc. Am., vol 65, p. 531, 1975.

[14] R. Maxwell, ERIM International, private communication.
Michael Hess received his MSEE from Stanford University in 1971. He worked in electro-optics technology at the Naval Air Warfare Center (formerly the Naval Air Development Center), Warminster, PA, before joining the Optical Sciences Division of the the Naval Research Laboratory in 1996. He has directed the development of several infrared technologies and airborne sensors, including forward-looking imaging sensors (FLIRs), focal plane arrays (FPAs), and search/track sensors (IRSTs). His primary work at the Naval Research Laboratory has been in the application of hyperspectral and polarization techniques to tactical sensor systems for enhanced detection of targets in clutter.

Richard Priest received his Ph.D. in physics from the University of Pennsylvania in 1972. Since joining the Optical Sciences Division of the Naval Research Laboratory in 1976, he has worked in several areas of applied optics, with concentration for the last 15 years in infrared technology. In this field, he has contributed to signature prediction, IR materials development and characterization, and system integration. He served on the program committee for the IRIS Targets, Backgrounds and Discrimination meeting for 10 years, three as Chairman. His current position is Head of the Theory Section, which hosts the low-background Infrared Range facility and associated reflectometers and scatterometers 